\title{
Colon Submucosal Microdialysis: A Novel in vivo Approach in Barrier Function Assessment - A Pilot Study in Rats
}

\author{
N. CIBIČEK ${ }^{1}$, H. ŽIVNÁ ${ }^{2}$, Z. ZADÁK ${ }^{3}$, J. KULÍŘ ${ }^{4}$, E. ČERMÁKOVÁ ${ }^{5}$ \\ V. PALIČKA ${ }^{1}$
}

${ }^{1}$ Institute of Clinical Biochemistry and Diagnostics, ${ }^{2}$ Radioisotope Laboratories and Vivarium, ${ }^{3}$ Research Laboratory of the Department of Gerontology and Metabolism, ${ }^{4}$ Department of Nuclear Medicine, ${ }^{5}$ Department of Biophysics and Biostatistics, Charles University in Prague, Faculty of Medicine in Hradec Králové and University Hospital Hradec Králové, Czech Republic

Received April 11, 2006

Accepted August 30, 2006

On-line available December 19, 2006

\begin{abstract}
Summary
During shock, prognosis of a patient depends largely on intestinal barrier function. The potency of gut epithelium to represent an obstacle to toxins is determined by the blood supply. All established methods of mucosal function determination necessitate the functional involvement of bloodstream. Microdialysis allows monitoring of extracellular substances in the gut submucosa, but its potential use for gut barrier integrity assessment is unknown. Twelve rats underwent perfusion of the descending colon either with $20 \%$ ethanol or control medium (vehicle). Both media contained equal amounts of a radioactive tracer substance $\left({ }^{51} \mathrm{Cr}\right.$-EDTA). Mucosal permeability for ${ }^{51} \mathrm{Cr}$-EDTA was assessed by microdialysate to luminal perfusate activity ratios. Sampling was performed using the colon submucosal microdialysis technique. The group subjected to ethanol treatment had profound macro- and microscopical alterations in perfused colonic segment associated with a significant increase in tracer permeability during ethanol exposure ( $2.354 \pm 0.298 \%$ for ethanol as opposed to $0.209 \pm 0.102 \%$ for control group, $\mathrm{p}<0.01$ ), which remained elevated for 60 min after cessation of ethanol administration (3.352 $\pm 0.188 \%$ for ethanol compared to $0.140 \pm 0.0838 \%$ for the control group, $\mathrm{p}<0.001)$. Submucosal microdialysis with radioactive tracer substance can be considered a feasible and advantageous alternative of gut barrier function estimation. Parallel monitoring of local tissue chemistry with this method remains a challenge in the future.
\end{abstract}

Key words

Microdialysis $\bullet$ Colon barrier function $\bullet{ }^{51} \mathrm{Cr}$-EDTA permeability $\bullet$ Rat

\section{Introduction}

In critical care, the prognosis of patients depends largely on intestinal mucosal barrier function being predominantly determined by a degree of blood supply
(Doig et al. 1998). Although an impressive array of previous studies proposes several experimental modalities of gut barrier integrity estimation (in the form of various clearance techniques), no approach has offered an indisputable capability to detect alterations in the course 
of ischemic periods, particularly if arterial clamping lasts for a longer time (Udassin et al. 1998, Iwata et al. 1998, Kawai et al. 1994). The principal problem lies in the requirement of ensuring constant transport of lowmolecular tracer between the lumen and the bloodstream regardless of its direction (Fihn et al. 2003). Although some procedures do not necessitate alterations due to repeated blood withdrawals (with or without compensatory fluid infusions), they may have other considerable impacts on the subject's physiology (e.g. detachment of kidneys by ligatures). In addition, when studying dynamics of extracellular molecules with short half-lives, frequent simultaneous sampling is needed. To our best knowledge, literature has not as yet suggested an approach allowing monitoring of intestinal permeability and related local metabolic processes (all without involvement of the bloodstream).

In many organs including the gut submucosa, microdialysis is a well-established means of observing the kinetics of extracellular substances (de la Pena et al. 2000, Kitano et al. 2000, Solligard et al. 2004). It is interesting that possibilities of bowel barrier function assessment have not yet been challenged using this advantageous technique. Hence, the aim of the present work was to develop a continuous method of mucosal permeability measurement based on a simple principle of passive tracer penetration from lumen into the submucosa and thus omitting the interfering involvement of the bloodstream (or urine) during ischemia. The study was designed to verify the hypothesis that microdialysis as a method applied in this region of the gut at least qualitatively responds to barrier integrity alterations according to our expectations. Our supposition was tested on a modified example of colon barrier impairment by ethanol (Sobue et al. 2003). There, we expected to find an increase in mucosal permeability represented by an elevation in radioactivity of the dialysate.

\section{Methods}

\section{Subjects}

Twelve conventionally bred adult white male rats of the Wistar strain (Biotest s.r.o., Konárovice, Czech Republic) weighing 250-450 g, were used. The animals were housed in the animal quarters for at least 7 days prior to the experiments at $22-24{ }^{\circ} \mathrm{C}, 40-60 \%$ relative humidity, air exchange 12-14 times per hour and 12-hour light-dark cycle periods. The rats had free access to standard laboratory rat chow pellets (ST 1-TOP, Velaz,
Prague, Czech Republic) except for 16-18 h before the experiments, when they fasted. During food deprivation, the animals were stationed in plastic cages with raised mesh floors to prevent coprophagia. Tap water was provided ad libitum until the day of the experiment. All animals received care in accordance with the guidelines set by the institutional Animal Use and Care Committee of the Charles University in Prague, Czech Republic. All experimental procedures were approved by the Committee for Protection of Animals against Cruelty (Charles University in Prague, Faculty of Medicine in Hradec Králové, Czech Republic).

\section{Substances}

For microdialysis, commercially available Ringer's solution (R1/1) (InMediec s.r.o., Luhačovice, Czech Republic) was used as a perfusion medium. It contained (in mmol.1 $1^{-1}$ ) $\mathrm{Na}^{+} 147.1, \mathrm{~K}^{+} 4.0, \mathrm{Ca}^{2+} 2.3, \mathrm{Cl}^{-}$ 155.6 (310 mOsm. $\mathrm{l}^{-1}$, room temperature). As luminal perfusate, a purchased solution of ${ }^{51} \mathrm{Cr}$-EDTA in 5 mmol. ${ }^{-1}$ EDTA, 433.64 MBq (11.72 $\left.\mathrm{mCi}\right)$ per $\mathrm{ml}$, pH 7.0 (Perkin Elmer, Boston, MA, USA), dissolved either in R1/1 (1: 1666.7 by volume), or in the same manner in a mixture of $\mathrm{R} 1 / 1$ and $96 \%$ ethanol (to obtain $20 \%$ ethanol solution), was employed. The former formula was followed to prepare a vehicle or control medium (CM), whereas the latter produced an ethanol medium (EM). Both media had the same ${ }^{51} \mathrm{Cr}$-EDTA concentrations and hence also ${ }^{51} \mathrm{Cr}$ activities given as cpm per volume unit. The radioactive solutions were prepared after delivery according to this protocol and were utilized without modifications in the course of all experiments regardless of their actual activities.

\section{Colon submucosal microdialysis technique}

All animals were anesthetized with single i.p. dose of pentobarbital (50 mg.kg ${ }^{-1}$, Nembutal ${ }^{\circledR}$, Abbott Laboratories, North Chicago, USA) and placed in a supine position on an unheated bed. They were kept under general anesthesia by cyclic i.p. administration of Nembutal $^{\circledR} \quad\left(15 \mathrm{mg} \cdot \mathrm{kg}^{-1} \cdot \mathrm{h}^{-1}\right)$. Body temperature was monitored using a rectal thermometer probe (Ama-digit ad $15^{\text {th }}$, Aprecision, Germany) and maintained at 38.2$38.8^{\circ} \mathrm{C}$ by means of a heating lamp. For all surgical procedures, clean, but not sterile instruments/materials were used. The trachea was carefully prepared and cannulated with a short polyethylene catheter (outer diameter $2 \mathrm{~mm}$ ) to ensure patent airways. 3-4 cm long midline laparotomy was performed with scissors through 
linea alba. Descending colon was exteriorized and, when necessary, the region in question was made free of formed stercus by gentle manipulation. When exposed, the organ was kept moist with gauze soaked in $0.9 \%$ saline (at room temperature). Firstly, parallel to the long axis of the organ at a distance of $5 \mathrm{~cm}$ from the anus, a 5$6 \mathrm{~mm}$ long tunnel was created from serosal aspect in its submucosal layer by means of a $28 \mathrm{G}$ needle. Probe position was selected so as to avoid interference with blood vessels. The instrument was run close under the serosa with care neither to penetrate through the mucosa into the colonic lumen nor to make an additional redundant opening in the serosa. Secondly, a microdialysis probe (MAB 1.2.4. with $6 \mathrm{kDa}$ cut-off polyethylenesulphone membrane, active length $4 \mathrm{~mm}$; outer diameter $0.24 \mathrm{~mm}$; Microbiotech/se AB, Stockholm, Sweden) was cautiously inserted into the preformed tunnel (Fig. 1a). The implantation technique was trained in advance on other animals and the exact localisation of the probe in the submucosal region of colon was verified histologically (Fig. 3a). Finally, the probe was fixed to the serosa at the tunnel entrance with an atraumatic Ethibond 5/0 suture (Ethicon ltd., U.K.). Throughout the experiment, continuous microdialysis was ensured by perfusing the catheters with R1/1 solution by means of a CMA 102 microdialysis pump (CMA Microdialysis AB, Solna, Sweden) at a perfusion rate of $1.5 \mu 1 . \mathrm{min}^{-1}$. For equilibration, an initial $40 \mathrm{~min}$ period (without specimen collection), followed by a yield of the first three control samples (during normal luminal perfusion) was designed. Sampling took place in $10 \mathrm{~min}$ intervals into $300 \mu \mathrm{l}$ polyethylene Beckman type vials. The specimens were stored at $-20{ }^{\circ} \mathrm{C}$ until analysis.

\section{Animal mode of colon luminal perfusion}

After successful implantation of a microdialysis probe, the oral part of the descending colon was ligated with a silk thread in a distance of 1-2 $\mathrm{cm}$ from the probe as close to the colonic wall as possible to avoid ischemization. Thereafter, a double-lumen cannula was inserted via anal route to permit continuous perfusion of the colonic lumen by means of a microprocessorcontrolled syringe pump (LD 20, Tesla Přelouč, Czech Republic). The inlet (inner) tubing ( $2.5 \mathrm{~cm}$ in length) was situated in the vicinity of the oral colonic ligature and was nearly as long as the perfused part of the colon. On the other hand, the outlet (outer) tubing was ligated on the opposite, aboral side of colon and had only minimal protrusion into the lumen $(0.5 \mathrm{~cm})$. After cannulation and

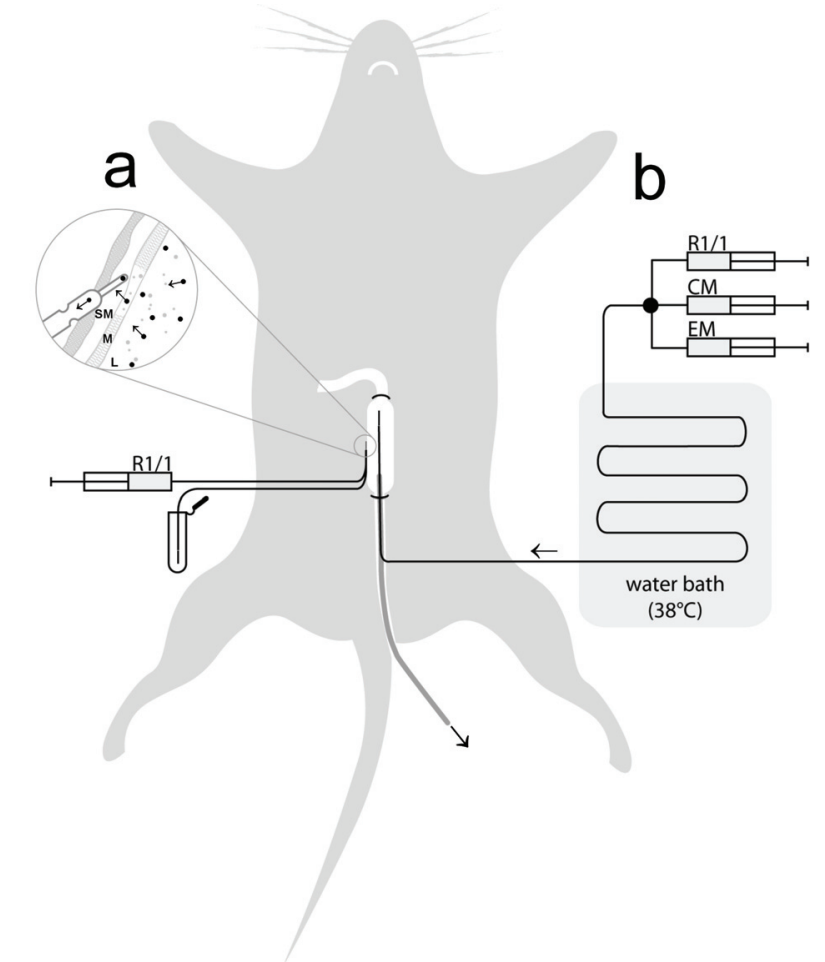

Fig. 1. A schematic view of in vivo submucosal microdialysis sampling (a) with parallel single-pass luminal perfusion of the descending colon (b) (details in the text). Note that this technique does not necessitate an artificial opening in the alimentary tract.

ligation was completed, a separated $3.0-3.5 \mathrm{~cm}$ long colonic lumen was prepared for single pass perfusion (Fig. 1b). At all times, to bring the temperature of the perfusion medium closer to body temperature, the inlet duct was passed in part through a thermostatic water bath $\left(38^{\circ} \mathrm{C}\right)$. For luminal washout, the flow was commenced at an initial rate of $25 \mathrm{ml} / \mathrm{h}$ for $30 \mathrm{~min}$ with $\mathrm{R} 1 / 1$ solution. In the next step, CM was applied using a three-way flow switch. Again, for quick washout, the same rate $\left(25 \mathrm{ml} . \mathrm{h}^{-1}\right)$ was used for $8 \mathrm{~min}$ followed by $2 \mathrm{~min}$ at the rate of $6 \mathrm{ml} \cdot \mathrm{h}^{-1}$. Thereafter, continuous microdialysis sample collection was commenced by first three 10-min control episodes (to obtain baseline levels). When perfusion with CM was finished and baseline specimens yielded, the flow was switched again for administration of EM. After washout period $\left(25 \mathrm{ml}^{-1}\right.$ for $8 \mathrm{~min}$ followed by $2 \mathrm{~min}$ at the rate of $6 \mathrm{ml}^{-1}$ ), three regular 10-min sampling intervals succeeded. When ethanol phase was completed, prior to the last, 60-min period of $\mathrm{CM}$ perfusion, a washout episode was carried out as described above. The total number of samples was 12 . During the whole procedure, care was taken not to allow air bubbles to enter into the perfusion system. To avoid 


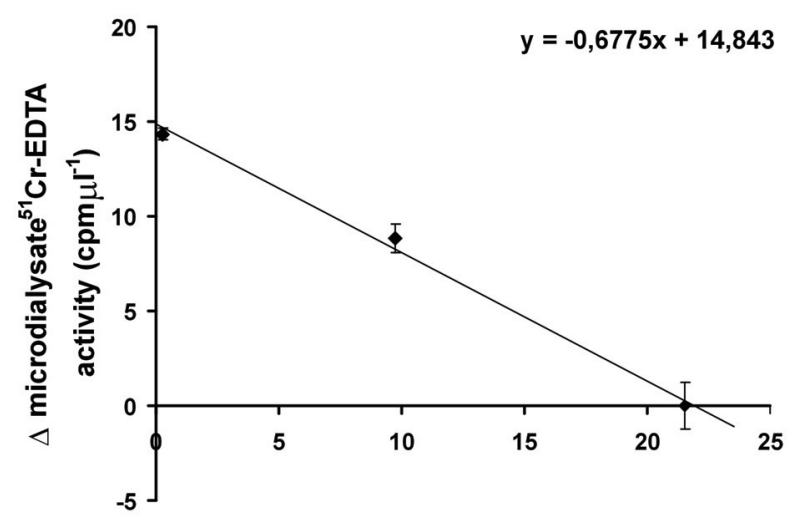

perfusate ${ }^{51}$ Cr-EDTA activity (cpm $\mu l^{-1}$ )

Fig. 2. In vitro probe calibration ("no net-flux" method). The $\Delta$ microdialysate (dialysate - perfusate) ${ }^{51} \mathrm{Cr}$-EDTA activity plotted against the perfusate ${ }^{51} \mathrm{Cr}$-EDTA activity gives a gradient representing the probe recovery $(67.8 \%)$. The $x$-intercept is in compliance with the actual activity of the surrounding medium. Results are means \pm S.E.M. of 6 measurements.

fluid losses and to ensure convenient i.p. administration of $\mathrm{Nembutal}^{\circledR}$, the skin layer of the abdominal opening was closed using microbulldog clamps.

\section{Experimental design}

The animals were allocated to two groups - the first $(\mathrm{C}$, control, $\mathrm{n}=6)$ group was examined as a sham group, i.e. without corrosive ethanol intervention (received only R1/1 and CM, with washouts preserved), whilst the second ( $E$, ethanol, $n=6$ ) group was exposed to a 30-min period of EM perfusion. All other procedures were identical for both groups. The animals were sacrificed by exsanguination from the abdominal aorta. After removal of the tubing from the descending colon and the isolation of the organ from the body, implanted probe was liberated, and colon tissue was dissected into a perfused and a proximal (oral) unperfused segment. The removed biological material was immersed in the preserving agent (10\% formalin) for ensuing histological analysis.

\section{Radioactivity (gamma decay) measurement}

A commercially available multi-crystal gamma counter LB 2111 (EG\&G Berthold, Germany) was utilized. The instrument was standardized for ${ }^{51} \mathrm{Cr}$ (gamma ray, $320 \mathrm{keV}$ ) and adjusted for energy levels ranging within $50-450 \mathrm{keV}$. Counts per minute (cpm) were calculated from 10-min measurements. For counting, the original plastic vials with collected samples (15 $\mu \mathrm{l})$ were used. To eliminate background counts,
24 identical vials with $15 \mu \mathrm{l}$ of $\mathrm{R} 1 / 1$ solution were assessed and the obtained mean value was subtracted from all measurements.

\section{Probe in vitro recovery calculation}

To obtain a basic knowledge of microdialysis probe function with regard to transport of ${ }^{51} \mathrm{Cr}$-EDTA via its semipermeable membrane, probe recovery was determined in vitro by no net-flux method as follows. Probe was immersed in $20 \mathrm{ml}$ of ${ }^{51} \mathrm{Cr}$-EDTA solution with specific activity of $21.53 \mathrm{cpm} . \mu \mathrm{l}^{-1}$ at standard laboratory temperature and perfused with three consecutive solutions (at equivalent temperature) of increasing activity $\left(0,9.73\right.$ and $\left.21.53 \mathrm{cpm} . \mu \mathrm{l}^{-1}\right)$. For equilibration, an initial 30-min period was allowed, which was succeeded by sampling in 10-min intervals into microvials. In each experiment, the surrounding medium was freshly prepared, perfusion rate set at $1.5 \mu 1 . \mathrm{min}^{-1}$ and six samples taken. The results were plotted on a graph and probe recovery was read from the regression equation (slope gradient).

\section{Barrier integrity determination}

To assess the function of colonic mucosal permeability, an updated ratio of activities was calculated using the following formula: [(probe perfusate cpm background cpm) / (luminal perfusate cpm - background cpm)] $\mathrm{x} 100$, where probe perfusate cpm is the activity obtained from $15 \mu \mathrm{l}$ microdialysis samples and luminal perfusate cpm is the mean number of counts given by six $15 \mu$ samples of luminal perfusate stock solution (CM or EM) shortly before administration to the animal. The results were expressed in $\%$.

\section{Histological analyses}

Colon biopsies were taken to evaluate the effect of ethanol perfusion at the microscopic level and for probe position verification. The samples of the descending colon were taken from both perfused and proximal (unperfused) segments. The tissues were fixed in $10 \%$ neutral buffered formalin and further treated according to standard procedures (hematoxylin-eosin stain). The sections were evaluated by light microscopic examination and photographed at 100-fold magnification.

\section{Statistics}

Data are expressed as means \pm S.E.M. For statistical evaluation, parametric (repeated measures ANOVA with multiple comparison by a post hoc Fisher's 

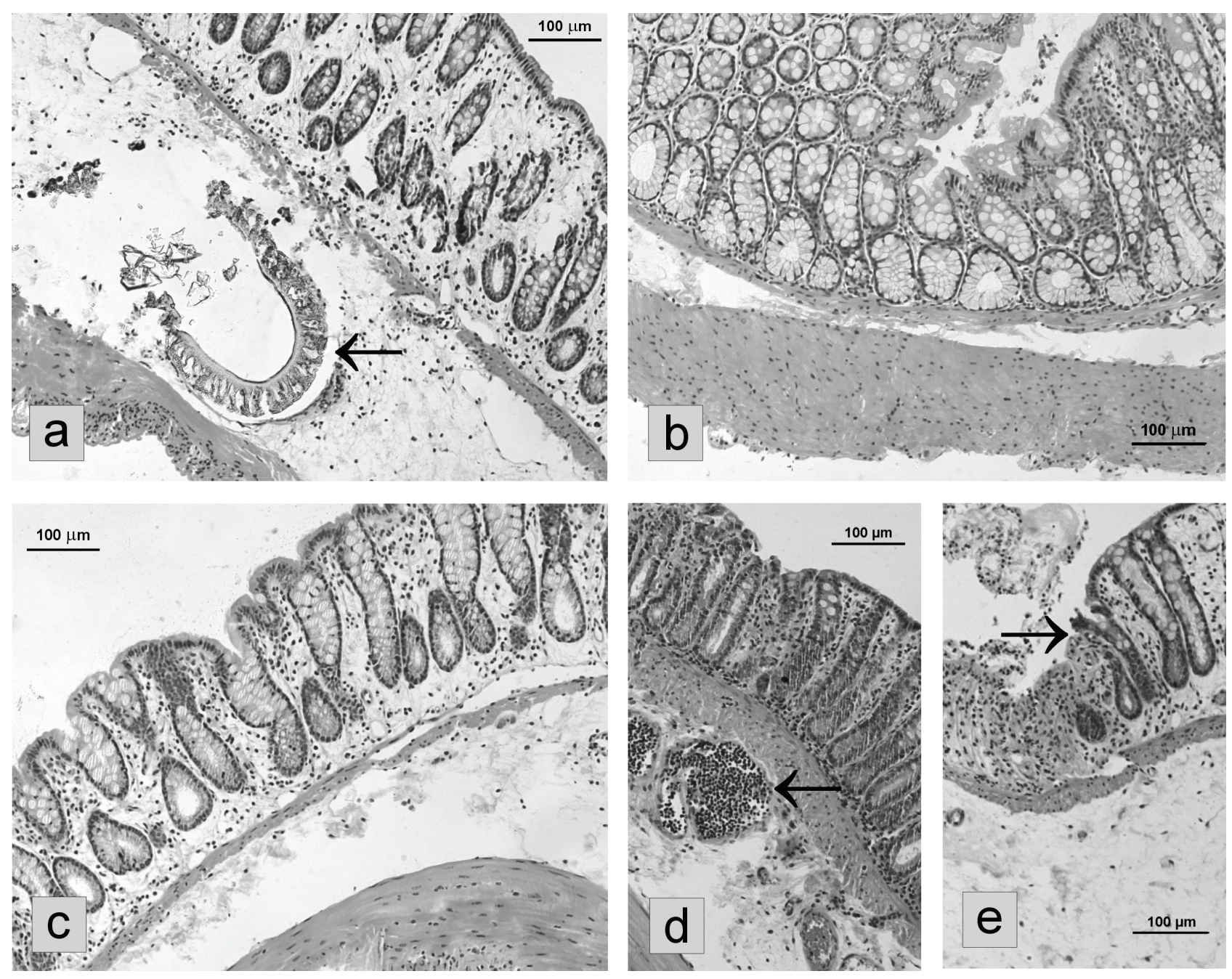

Fig. 3. Histological appearance of the wall of descending colon (HE stain, magnifications marked by scale bars). a, Probe position verification. Arrow points to probe remnants. The probe was damaged after experiment during tissue processing for slide preparation. b, Proximal (unperfused) part of colon showing intact tissue layers. c, Picture taken after the organ's luminal perfusion with vehicle alone (control group). No marked changes observable. d, and e, Ethanol group. Leukocyte-filled dilated submucosal vessels (arrow, d) accompany the loss of mucosal architecture (arrow, e) owing to erosive action of ethanol.

LSD test) and non-parametric (Friedman's ANOVA with Wilcoxon's test) methods were applied. The data were processed by the programs NCSS 2004 and Statistica. The chosen level of significance was $\alpha=0.05$.

\section{Results}

\section{Histology}

Microdialysis probes were positioned exactly within the submucosal layer of colonic wall, as indicated in Figure 3a. The unperfused part of the colon was unaffected by the experimental procedure (Fig. 3b). Application of R1/1 and vehicle (CM) had no material influence on the microscopical picture of the perfused part of the gut (Fig. 3c). However, after administration of ethanol, marked submucosal reaction/impairment was confirmed by histology (Fig. 3d,e).

\section{Microdialysis data}

The results of probe calibration are depicted in Figure 2. The in vitro recovery of the used probe type under the given conditions was estimated to be $67.8 \%$. With regard to barrier integrity estimation by microdialysis, there were some doubts as to the normality of data distributions given by the low number of subjects. However, non-parametric tests confirmed the findings of parametric methods. During the administration of $20 \%$ ethanol, mucosal tracer permeability showed significant elevations $(2.354 \pm 0.298 \%$ for ethanol as opposed to $0.209 \pm 0.102 \%$ for control group, time interval $60 \mathrm{~min}$, $\mathrm{p}<0.01$ ), which persisted or were even more pronounced after the ethanol instillation was discontinued until the end of the experiment, i.e. for at least $60 \mathrm{~min}(3.352 \pm 0.188 \%$ for ethanol compared to $0.140 \pm 0.084 \%$ for the control group, time interval $120 \mathrm{~min}, \mathrm{p}<0.001$ ) (Fig. 4). 


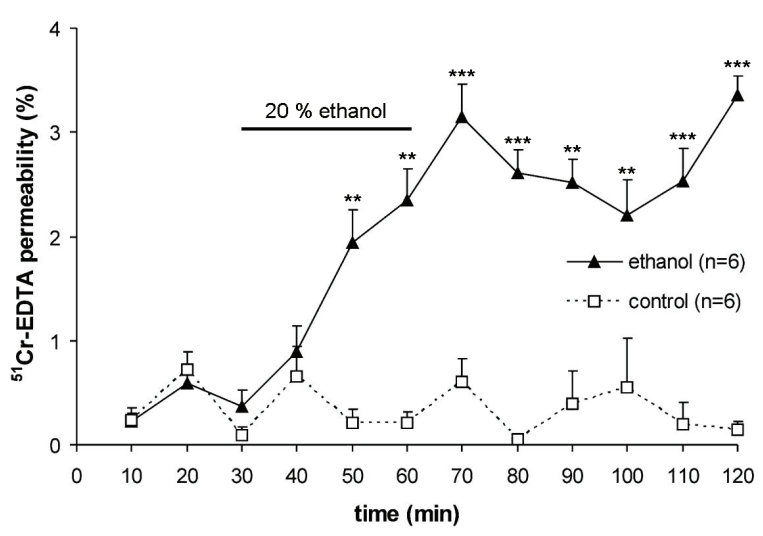

Fig. 4. Colon barrier function as assessed by lumen-tosubmucosa ${ }^{51} \mathrm{Cr}$-EDTA permeability $(15 \mu \mathrm{l}$ perfusion medium to $15 \mu \mathrm{l}$ microdialysate activity $\times 100$, expressed in \%). Note the gradual increase in mucosal permeability in the course of ethanol perfusion in ethanol group. Results are means + S.E.M. At shown intervals, markings $* *$ and $* * *$ denote $\mathrm{p}<0.01$ and $\mathrm{p}<0.001$, respectively.

\section{Discussion}

\section{Methodological considerations}

${ }^{51} \mathrm{Cr}$-EDTA was employed as a suitable tracer because of its low molecular weight $\left(352 \mathrm{~g} . \mathrm{mol}^{-1}\right)$ and diameter $(6.8 \AA)$, i.e. characteristics that account for its free and rapid movement via vascular or endothelial barriers (Nylander et al. 1989). Hence, the only determinant of ${ }^{51} \mathrm{Cr}$-EDTA mucosal permeability is epithelial lining integrity (function of tight junctions). In this context, microdialysis probes generally imitate blood vessels. Providing an adequate molecular diameter/pore cut-off ratio, perfusion rate and good function, probes generally represent a moderate and determinable obstacle to tracer diffusion as was also the case in our experiment. Moreover, application of ${ }^{51} \mathrm{Cr}$-EDTA was advantageous, since it contains a radiolabeled atom. Measurements of gamma-activity do not require any biochemical assays with inevitable sample losses, whereby they may enable serial determination of other substances, e.g. metabolic markers or pharmaceuticals. In this way, twice as much information could be yielded from one approach in general and from microdialysis, as a method coping with low sample volumes, in particular. Unfortunately for present time, we were not able to directly demonstrate this potential unique capacity of microdialysis due to sample loss during secondary analytical procedure. Unlike elsewhere, our perfusion solutions were prepared once and used throughout the whole experiment. Hence, to eliminate the effect of radioactive decay and loss of
${ }^{51} \mathrm{Cr}$ activity (half-life $=27.7$ days) on results of mucosal permeability, the approach of using a ratio of cpm values was chosen.

\section{The effect of ethanol perfusion}

The lack of change in the studied parameter within control group indicates that the implantation technique and presence of the probe in the submucosal region did not affect barrier integrity. The marked increase in ${ }^{51} \mathrm{Cr}$-EDTA permeability in the ethanol group was consistent with observed macro- and microscopic mucosal injury and leukocyte infiltration (Fig. $3 \mathrm{~d}$ and e, macroscopic picture not shown). These findings convincingly showed a profound disintegration of colonic epithelial barrier due to 30-min treatment with $20 \%$ ethanol. It is notable that an analogous protocol applied in the stomach produced a significant, yet transient increase, followed by restitution in ${ }^{51} \mathrm{Cr}$-EDTA blood-to-lumen clearance after $10 \mathrm{~min}$ perfusion with $20 \%$ ethanol. Here, the finding of rapid barrier recovery was confirmed by minimal histological damage to the mucosa (Sobue $e t$ al. 2003). However, a single-shot intracolonic administration of $50 \%$ ethanol caused elevations in ${ }^{51} \mathrm{Cr}$-EDTA lumento-blood clearance, which persisted at least for $3 \mathrm{~h}$, but would completely resolve by 2 days thereafter (Stein $e t$ al. 1998). Likewise, in a comparable model of colitis induced by trinitrobenzene sulfonic acid in $30 \%$ ethanol, the most marked enhancement in neutrophil infiltration of colon (as estimated by myeloperoxidase activity and confirmed by histology) occurred 3-6 $\mathrm{h}$ after the induction of colitis. During this period, the greatest increase in colonic permeability to ${ }^{51} \mathrm{Cr}$-EDTA was also observed (Wallace et al. 1992). To sum up, concerning intraluminal ethanol perfusion, the results obtained from microdialysis applied in the vicinity of tight junctions conform to the outcomes of clearance methods. Nevertheless, in order to demonstrate the suggested basic advantage of proposed technique, a parallel comparison with an established method (based on a non-radioactive tracer) should be performed on an ischemic model in the future.

\section{Conclusions}

The most important finding of our study was a significant elevation of tracer penetration into the microdialysis probe following the administration of ethanol. The proposed methodology was capable of detecting barrier injury without necessitating organ detachments, blood withdrawals or even vessel 
catheterization. This pilot study provides a basement for development and introduction of a novel method for a wide range of experimental settings involving mucosal permeability measurements. The presented results indicate that submucosal microdialysis can be considered a feasible and advantageous alternative of gut barrier function estimation. Parallel monitoring of local tissue chemistry or pharmacology with this method remains a future challenge.

\section{Acknowledgements}

The authors wish to acknowledge a helpful and obliging cooperation with Ms. Kriesfalusyová in the Radioisotope Laboratory. We also thank Ms. Hollerová for skillful technical assistance with preparation of histological slides and Assoc. Prof. Rudolf for making electronical photographs. This work was principally supported by a Research Project from the Czech Ministry of Foreign Commerce MZO 00179906.

\section{References}

De la PENA A, LIU P, DERENDORF H. Microdialysis in peripheral tissues. Adv Drug Deliv Rev 45: 189-216, 2000.

DOIG CJ, SUTHERLAND LR, SANDHAM JD, FICK GH, VERHOEF M, MEDDINGS JB: Increased intestinal permeability is associated with the development of multiple organ dysfunction syndrome in critically ill ICU patients. Am J Respir Crit Care Med 158: 444-451, 1998.

FIHN BM, SJOQVIST A, JODAL M: Involvement of enteric nerves in permeability changes due to deoxycholic acid in rat jejunum in vivo. Acta Physiol Scand 178: 241-250, 2003.

IWATA F, JOH T, UEDA F, YOKOYAMA Y, ITOH M: Role of gap junctions in inhibiting ischemia-reperfusion injury of rat gastric mucosa. Am J Physiol 275: G883-G888, 1998.

KAWAI T, JOH T, IWATA F, ITOH M: Gastric epithelial damage induced by local ischemia-reperfusion with or without exogenous acid. Am J Physiol 266: G263-G270, 1994.

KITANO M, NORLÉN P, HÅKANSON R: Gastric submucosal microdialysis: a method to study gastrin- and foodevoked mobilization of ECL-cell histamine in conscious rats. Regul Pept 86: 113-123, 2000.

NYLANDER O, KVIETYS P, GRANGER DN: Effects of hydrochloric acid on duodenal and jejunal mucosal permeability in the rat. Am J Physiol 257: G653-G660, 1989.

SOBUE M, JOH T, OSHIMA T, SUZUKI H, SENO K, KASUGAI K, NOMURA T, OHARA H, YOKOYAMA Y, ITOH M: Contribution of capsaicin-sensitive afferent nerves to rapid recovery from ethanol-induced gastric epithelial damage in rats. $J$ Gastroenterol Hepatol 18: 1188-1195, 2003.

SOLLIGÅRD E, JUEL IS, BAKKELUND K, JOHNSEN H, SAETHER OD, GRONBECH JE, AADAHL P: Gut barrier dysfunction as detected by intestinal luminal microdialysis. Intensive Care Med 30: 1188-1194, 2004.

STEIN J, RIES J, BARRETT KE: Disruption of intestinal barrier function associated with experimental colitis: possible role of mast cells. Am J Physiol 274: G203-G209, 1998.

UDASSIN R, HASKEL Y, SEROR D, WELBOURNE TC: Plasma-to-lumen clearance of para-aminohippurate can replace ${ }^{51} \mathrm{Cr}$-EDTA clearance in the evaluation of intestinal mucosal injury. Pediatr Surg Int 13: 112-114, 1998.

WALLACE JL, HIGA A, MCKNIGHT GW, MACINTYRE DE: Prevention and reversal of experimental colitis by a monoclonal antibody which inhibits leukocyte adherence. Inflammation 16: 343-354, 1992.

\section{Corresponding author}

N. Cibiček, Institute of Clinical Biochemistry and Diagnostics (ÚKBD), University Hospital (FN), Sokolská 581, 500 05 Hradec Králové, Czech Republic. E-mail: cibicek@seznam.cz 